\title{
Smell, Taste, and Temperature Interfaces
}

\section{JAS BROOKS}

University of Chicago, Chicago, United States

PEDRO LOPES

University of Chicago, Chicago, United States

JUDITH AMORES

MIT Media Lab and MGH/HMS, Cambridge, United States

EMANUELA MAGGIONI

University College London, London, United Kingdom

HARUKA MATSUKURA

Osaka University, Toyonaka, Japan

MARIANNA OBRIST

University College London, London, United Kingdom

ROSHAN LALINTHA PEIRIS

Rochester Institute of Technology, Rochester, United States

NIMESHA RANASINGHE

University of Maine, Orono, United States

Everyday life hinges on smell, taste, and temperature-based experiences, from eating to detecting potential hazards (e.g., smell of rotten food, microbial threats, and non-microbial threats such as from hazardous gases) to responding to thermal behavioral changes. These experiences are formative as visceral, vital signals of information, and contribute directly to our safety, well-being, and enjoyment. Despite this, contemporary technology mostly stimulates vision, audition, and - more recently - touch, unfortunately leaving out the senses of smell taste and temperature. In the last decade, smell, taste, and temperature interfaces have gained a renewed attention in the field of Human Computer Interaction, fueled by the growth of virtual reality and wearable devices. As these modalities are further explored, it is imperative to discuss underlying cultural contexts of these experiences, how researchers can robustly stimulate and sense these modalities, and in what contexts such multisensory technologies are meaningful. This workshop addresses these topics and seeks to provoke critical discussions around chemo- and thermo-sensory HCI.

CCS CONCEPTS • Hardware $\sim$ Emerging technologies $\sim$ Emerging interfaces $•$ Human-centered computing $\sim$ Human computer interaction (HCI)

Additional Keywords and Phrases: Smell, taste, temperature, chemesthesis 


\section{ACM Reference Format:}

First Author's Name, Initials, and Last Name, Second Author's Name, Initials, and Last Name, and Third Author's Name, Initials, and Last Name. 2018. The Title of the Paper: ACM Conference Proceedings Manuscript Submission Template: This is the subtitle of the paper, this document both explains and embodies the submission format for authors using Word. In Woodstock '18: ACM Symposium on Neural Gaze Detection, June 03-05, 2018, Woodstock, NY. ACM, New York, NY, USA, 10 pages. NOTE: This block will be automatically generated when manuscripts are processed after acceptance.

\section{BACKGROUND}

Contemporary interactive devices mostly stimulate only our vision, hearing, and - only more recently - touch. In stark contrast to the limited sensory input and output of modern computers, human chemo- and thermo-sensations are both essential parts of our everyday lives. The sense of smell is what allows us to understand a wide range of everyday experiences, from pleasurable ones (good food) to detecting potential hazards (e.g., smell of rotten food or hazardous gases) [26]. Our sense of taste contributes to our understanding of flavor during consumption, can embody affective states [10, 19], and affect human behavior [28]. Finally, our sense of temperature contributes to concepts of social proximity [13] and also impacts human behavior (e.g. through behavioral thermoregulation [9]).

Attempts at redesign the interface I/O capabilities to acknowledge the very chemical, reactive interactions humans are afforded have been - historically - very isolated endeavors. Early attempts in the scent technology were propelled by cinema's desire to control the atmosphere of the movie theater, with air conditioning and ventilation to remove the outside world's influence on sales (e.g. muggy hot days) or the smells of other moviegoers in the audience [7]. This atmospheric conditioning led researchers such as Hans Laube (Smell-O-Vision [15]) and Charles Weiss (AromaRama) to consider also new possibilities; rather than deodorizing a space, they proposed: scenting it. Simultaneously, in the sphere of gastronomy, the flavor industry labored over methods to objectively determine, measure, and then adjust the sensory qualities of food. Acknowledging flavor as a multisensory phenomenon, food researchers integrated both new instruments and trained taster panels for food quality testing [3], combining the unique affordances of both chemical analysis and human perception. Stories such as Frank Felitta's Substance X [29] or Roald Dahl's Charlie and the Chocolate Factory [8] imagined the extremes of such food and technology sciences, with systems that could produce any flavor to the detriment of their consumer. Yet this is technically a complex road for engineering, as it took nearly 40 years before a hand-held device could re-produce simple flavors, the food simulator [14]. Finally, researchers in the $20^{\text {th }}$ century had explored the incorporation of thermal feedback for experiences with limited capabilities, such as in Morton Heilig's Sensorama system [12].

\section{THE GROWTH OF SMELL, TASTE, AND TEMPERATURE INTERFACES FROM 2000-2020}

Over the last two decades, a noticeable increase in engineering and understanding these smell, taste and temperature interfaces can be traced within the field of Human-Computer Interaction [1, 2, 18, 22, 24, 25]. To illustrate these trends, we aggregated metadata from Crossref for all journal and proceedings articles published by the Association for Computing Machinery (ACM) and the Institute of Electrical and Electronics Engineers (IEEE) using the rcrossref package [6] with metadata collected as of October 8, 2020. To collect papers, we used the following queries: electronic nose, olfactory, odor, scent, perfume, and smell; gustatory, flavor, food, cooking, and eating; temperature feedback and thermal feedback; and, multisensory and mulsemedia (MULtiple SEnsorial MEDIA). As depicted in Figure 1, smell- and taste- related articles have seen stark increases in publication rates, with spikes after the 2000s. Temperature-related articles had a 
steady interest in the $20^{\text {th }}$ century, followed by an increase in published articles starting the mid-2000s. Finally, mulsemedia-related research has slowly increased since the 2000 s.
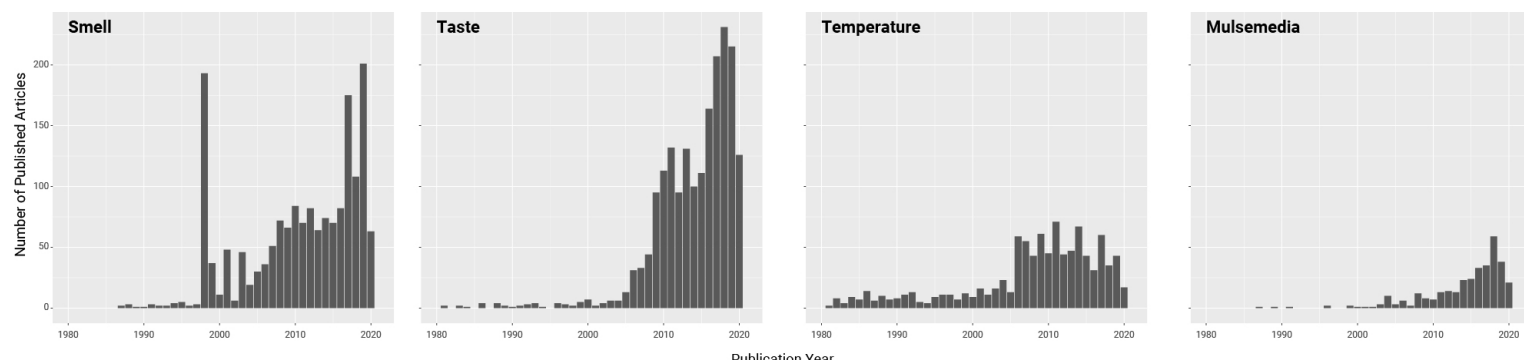

Figure 1: Bar graphs showing publication trends for smell-, taste-, temperature-, and mulsemedia related publications published by the ACM or IEEE since the 1980 s. All four subjects have seen steady rises since the 2000 s.

However, the last workshop related to these topics was held 5 years ago at CHI'16 by Obrist et al. [20]. Yet since this pioneer effort at $\mathrm{CHI}$, the field has kept expanding, as depicted by our literature analysis-this marks a need to gather this community again and discuss new challenges, technologies, and recent advances.

In fact, with the growth in immersive environments (VR) and wearable devices, smell/temperature/olfaction interfaces have been reshaped and introduced to new domains. For instance, olfactory interfaces are now incorporated into sleep and dream research [5]. Matsukura and Ishida proposed and explored the development of interfaces to amplify human olfaction [18]. New interfaces have been developed for thermal feedback [22] and virtually simulating wetness [11], a sensation we have no dedicated receptors for. Research on flavor simulation and modification has continued, incorporating concepts of gustatory augmentation [23] or food printing to modulate satiety [16]. Finally, research is emerging on leveraging human chemesthesis (actuation of the trigeminal nerve) for computer interfaces [4]. As such, this workshop will bring together researchers and practitioners from academia, industry, and arts to discuss and share their research and insight.

(a) smell

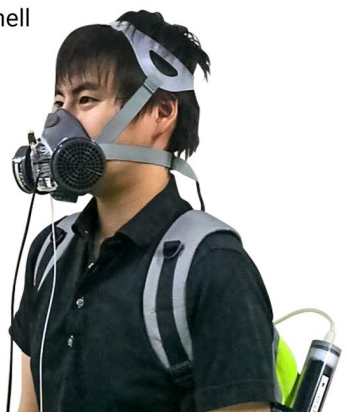

(b) taste

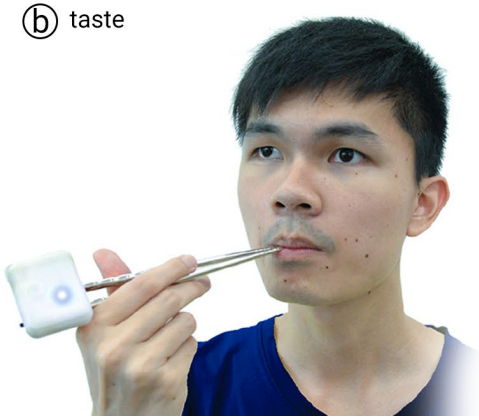

(C) temperature

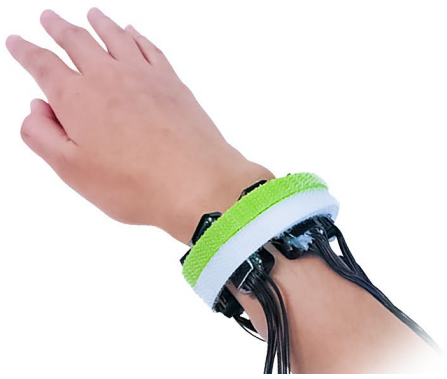

Figure 2: The Smell, Taste, and Temperature workshop covers chemo- and thermo-sensory interfaces. Above are three examples of such devices: (a) The Olfactory Assist Mask is a system that makes trace chemical gases interpretable to humans by substituting it with smellable odors [17]. (b) Ranasinghe et al. explored augmenting flavor by adding digital gustation to utensils like chopsticks

[23]. (c) The ThermalBracelet is a device which provides fast-switching thermal feedback to the wrist using groups of four thermoelectric elements [21]. 


\subsection{Topics of Interest}

Chemo- and thermo-sensory interfaces have unique challenges and opportunities, such as the challenge of developing interfaces while the basic science behind the sensations are an open problem in psychology, biology, and neuroscience (e.g. in smell research). The topics of interest for the workshop incorporate and expand upon those from the CHI'16 workshop, and include, but are not limited to, the following

- Design and use of multi-sensory technologies.

- Technologies pushing forward smell, taste, and thermal experiences.

- Chemo- and thermo-sensory augmentation.

- Cultural aspects and contexts of multi-sensory interactions in everyday life and history. We need to think about the contexts of these experiences which influence and shape both the technologies being developed and their societal adoption.

- $\quad$ Techniques \& recommendations for recording and stimulating chemo- and thermo-sensations.

We aim to additionally highlight and discuss open challenges in the field, which include, but are not limited to,

1. Power consumption: affects all chemo- and thermo-sensory devices, but especially thermo-.

2. Chemical: affects all chemo-sensory devices (smell, taste, chemesthetic), often non-reducible, recordings and replay of experiences, precision, inter- and intra-modality effects (e.g. suppression effects).

3. Non-technical: complexity of dimensions, precision, cultural adoption, sharing data across HCI researchers, open sourcing of devices, simultaneously ongoing basic scientific research.

\subsection{Goals \& Rationale}

The goal of our workshop is four-fold: (1) to gather researchers and practitioners who are conducting research at the intersection of technology and chemo- or thermo-sensation, as well as build a network amongst the community; (2) discuss recent trends and challenges of current research; (3) promote the research of smell, taste, and temperature interfaces to larger, international audiences; and (4) conceive plans for future events.

We think this workshop is timely as (1) premiere conferences such as CHI'20 did not happen, which means there was a one-year gap in sharing new devices and techniques from this community. (2) The last time such a workshop was organized was five years ago [20] (focused also on "touch" interactions, which ours does not focus on); many advances have since been made and many new researchers are joining the field. (3) To our surprise, we found that this field is blooming: in response to CHI'20 not happening, we self-organized an online symposium with 255 people registered from over 33 countries across the world, with 152 people tuning in for the Smell Session, 93 for the Temperature Session, and 78 for the Taste Session.

\section{ORGANIZERS}

We provide short biographies on each workshop organizer. The organizers' experiences are complementary, providing a wide range of perspectives on the workshop's topic. The lead author has collaborated with the organizers on the workshop's past iteration ( $\underline{\text { STT'20 }}$, an online symposium for Smell, Taste, and Temperature papers published at CHI'20).

Jas Brooks is a PhD student in the Department of Computer Science at the University of Chicago. Jas focuses on computer interfaces that directly modulate human chemosensation, such as smell, taste, and chemesthesis. Their most recent device leveraged chemical stimulation of the nose's trigeminal nerve endings to induce temperature illusions. Jas's research is supported by a National Science Foundation Graduate Research Fellowship and has been covered by media publications such as IEEE Spectrum. Website: jasbrooks.net 
Pedro Lopes is an Assistant Professor in Computer Science at the University of Chicago. Pedro focuses on integrating computer interfaces with the human body-exploring the interface paradigm that supersedes wearable computing. Some of these new integrated-devices include: a device based on muscle stimulation that allows users to manipulate tools they never seen before or that accelerate their reaction time, or a device that leverages the nose to create an illusion of temperature. Pedro's work also captured the interest of media, such as New York Times or NewScientist, and was exhibited at Ars Electronica and the World Economic Forum. Website: lab.plopes.org

Judith Amores is a Research Fellow at the MGH/Harvard Medical School and a Research Affiliate at the MIT Media Lab, where she did her $\mathrm{PhD}$ and master's and helped run VR/AR at MIT as a co-president. She holds a multimedia engineering degree and has worked at Microsoft Research, URL Barcelona, and the Google Creative Lab. Her awards and publications include over 27 peer-reviewed research papers, two patents, a Facebook Graduate Fellowship, LEGO Foundation sponsored research, and was a finalist of the Innovation by Design Awards. She also received the Scent Innovator Award by CEW and IFF. Website: judithamores.com

Emanuela Maggioni is a Research Fellow at the University College London. Emanuela joined the SCHI Lab in February 2016 and is working on multisensory perception. She is passionate about olfaction and the design of olfactory interfaces. She received a PhD in Social, Cognitive, and Clinical Psychology in 2015. She was a visiting researcher at the University of Oxford and UCL. She is interested in understanding how multisensory interactions/experiences, with particular attention to olfactory stimulations, affect emotions and decision-making process from both theoretical and practical perspectives, using behavioral and physiological based methodologies. Recently, Emanuela was awarded the Royal Academy of Engineering (RAEng) Enterprise Fellowship 2019-2020. Website: multi-sensory.info

Haruka Matsukura is an Assistant Professor at Osaka University's Graduate School of Engineering Science. She completed her $\mathrm{PhD}$ at the Tokyo University of Agriculture and Technology in 2013 and was employed by Osaka University as an Assistant Professor in 2017. She was a JSPS Fellow (DC1) from 2010 to 2013. Haruka's work includes the Smelling Screen and the Olfactory Assist Mask. Her research interests include olfactory displays, gas source localization robots, and body extension. Website: haruka-matsu.com

Marianna Obrist is Professor of Multisensory Interfaces at UCL, Department of Computer Science. She has established the SCHI Lab, an interdisciplinary research group, investigating touch, taste, and smell as interaction modalities. She is a co-founder of OWidgets Ltd, a University start-up developing novel software and hardware solutions for smell experience design. She is an inaugural member of the ACM Future of Computing Academy and was selected Young Scientist 2017 and 2018 to attend the World Economic Forum. She is a Visiting Professor at the Royal College of Art and has recently published a book on Multisensory Experiences: where the senses meet technology. Website: multisensory.info

Roshan Peiris is an Assistant Professor at the School of Information at the Rochester Institute of Technology. He currently leads the Altered Reality research group that focuses on understanding the human perception to create new technologies that can alter and enhance our experiences. His research areas include haptics, multisensory mixed reality technologies, wearable computing, and accessibility. Roshan received his $\mathrm{PhD}$ from the National University of Singapore in Integrative Sciences and Engineering and his BSc in Electrical Engineering from the University of Moratuwa. His work includes ThermoVR, ThermalBracelet, LiquidReality, and LiquidVR, and has received several awards at international events. Website: roshanpeiris.com

Nimesha Ranasinghe is an Assistant Professor at the School of Computing and Information Science and directs the Multisensory Interactive Media lab (MIM lab) at the University of Maine. He completed his PhD at the Department of Electrical and Computer Engineering, National University of Singapore (NUS) in 2013. Dr. Ranasinghe's research 
interests include Multisensory Interactive Media, Human-Computer Interaction, Augmented and Virtual Reality. He is well-known for his Digital Lollipop (a.k.a. Virtual Flavors) and Virtual Cocktail (Vocktail) inventions and featured in numerous media publications worldwide, including New Scientist, New York Times, Time Magazine, BBC Radio, Discovery Channel, and Reuters. Website: mimlab.info

\section{WORKSHOP STRUCTURE}

The one-day workshop will be organized according to CHI 2021's format, which depends on progress of COVID-19 restrictions. Our structure enables us to organize both hybrid or virtual, depending on restrictions and enabling a wide participation.

In either hybrid or virtual format, our workshop consists of a synchronous meeting with panels, provocation talks to spark discussion, and in-depth breakout discussions. In case of a fully virtual CHI'20, each speaker's provocation talk can be provided as a prerecorded video, and participants are asked to provide short video presentations of their submission, which would then be distributed ahead of time. The estimated number of virtual participants is 20-30. Based on our experience from the self-organized sessions in 2020 (with 255 registrations), we expect to fill out these seats.

\subsection{Website}

The workshop webpage can be found at stt21.plopes.org. The website will promote the workshop theme, and will include background information on the topic, Call for Participation, workshop schedule, backgrounds of each of the organizers, and links to relevant papers and news. Additionally, accepted applications will be publicly available for download alongside, later, the asynchronous paper presentation videos.

\subsection{Pre-Workshop Plans}

The workshop's Call for Participation will be distributed via mailing lists (e.g. ACM SIGCHI, UIST) to researchers in the field of HCI, UX, and related areas interested in multisensory research. The call will additionally be distributed to all the 255 registered participants of our self-organized 2020 event, and to art and online organizations (e.g. the Institute for Art and Olfaction, Aroma Discord, the Association for the Study of Food and Society). Participant videos will be available on the website so that everyone can get to know each other before the workshop begins.

To summarize important dates for the workshop, we gather them below:

- Final submission deadline: Friday, February 19, 2021 at 12:00pm (noon) PT.

- Notification date: Friday, March 12, 2021 at 12:00pm (noon) PT.

- Video deadline: Friday, April 23, 2021 at 12:00pm (noon) PT.

\subsection{Activities and Schedule}

The one-day workshop is split into three brainstorms featuring provocation talks by organizers followed by discussions. Provocation talks are short presentations meant to reframe the current trends or future possibilities with an organizer's evocative vision. The exact times of these brainstorms will be scheduled according to participants, in consideration of varying time zones. For sake of clarity, we present the schedule as if it began at 9:00am.

- 09:00 - 9:10: Introduction and welcome by Jas Brooks and Pedro Lopes.

- 09:10 - 10:30: Brainstorm 1, Future of Smell Interfaces

○ 30 minutes: 10-minute provocations by Haruka Matsukura, Emanuela Maggioni, and Judith Amores. 
○ 20 minutes: Breakout discussions in groups of 4-5 participants with 1-2 organizers.

- 20 minutes: Reconvene to discuss the challenges and possibilities of smell interfaces.

- $10: 30$ - 11:00: Break

- 11:00 - 12:30: Brainstorm 2, Future of Taste Interfaces

- 20 minutes: 10-minute provocations by Marianna Obrist and Nimesha Ranasinghe.

- 20 minutes: Breakout discussions in groups of 4-5 participants with 1-2 organizers.

- 20 minutes: Reconvene to discuss the challenges and possibilities of taste interfaces.

- $\quad$ 12:30 - 14:00: Break

- 14:00 - 15:30: Brainstorm 3, Future of Thermal and Chemesthetic Interfaces

- 20 minutes: 10-minute provocations by Roshan Peiris and Jas Brooks.

- 20 minutes: Breakout discussions in groups of 4-5 participants with 1-2 organizers.

- 20 minutes: Reconvene to discuss the challenges and possibilities of thermal and chemesthetic interfaces.

- 16:00 - 17:00: Closing discussion

- Zooming out to think about the long-term vision for Smell, Taste, and Temperature Interfaces alongside plans for the next meeting and exchanging contacts to foster collaboration.

- 17:00: End of Workshop

- 17:20 - 18:00: Optional social event, Virtual Screenings with Scratch and Sniff cards

- Ahead of the workshop, participants and organizers will receive scratch and sniff cards for the virtual screening of scented short films.

○ Special Guest: Tammy Burnstock, artist who lead the olfactory restoration of A Tale of Old Whiff.

\subsection{Post-Workshop Plans}

All accepted submissions to the workshop, asynchronous video presentations, and provocation talks will be made public on the website and via YouTube. Authors of the submissions will be invited to send updates on follow-up-studies related to the workshop submissions. In accordance with the authors, these updates will also be published on the website. Furthermore, the outcome of the workshop will be submitted as a summary report to ACM Interactions to reach out to a larger community of academics and practitioners interested in new frontier research in HCI. To additionally encourage interest, outcomes and updates will be shared across online communities, such as HCI Facebook groups (e.g. CHI Meta, MPHCI\&UX, ACM SIGCHI), media art groups (e.g. The New Media Thing), and multisensory-specific groups (e.g. Aroma Discord, the Institute for Art and Olfaction, the Association for the Study of Food and Society).

\section{CALL FOR PARTICIPATION}

The "Smell, Taste, and Temperature Interfaces" workshop addresses the burgeoning subfield of chemo- and thermosensory technologies and experiences. This one-day workshop will offer an interdisciplinary forum of discussion for academics and practitioners interested in leveraging these sensations.

This workshop focuses on the challenges of chemo- and thermo-sensory interfaces (smell, taste, and temperature) as well as their cultural contexts, usage, and resulting experiences.

Researchers and practitioners from academia, arts, and industry are invited to apply to the workshop by submitting a 1-4 page position or research paper in the ACM Master Article Submission Template single column format via the Easy Chair system (see workshop webpage). The submission deadline is Friday, February 19, 2021 at 12:00pm (noon) PT. 
All applications will be reviewed by the workshop organizers and selection will be based on the paper's quality. Applicants will be notified of decisions on Friday, March 12, 2021 at 12:00pm (noon) PT. Accepted papers will be made available at the workshop webpage. Upon acceptance, participants are asked to produce a 5-minute introduction/paper presentation video, which will also be shared publicly on the workshop website. At least one author of each accepted paper must attend the workshop. All participants must register for both the workshop and for at least one day of the conference.

Further details can be found on the workshop webpage: stt21.plopes.org.

\section{REFERENCES}

[1] Altarriba Bertran, F. et al. 2018. Visualising the Landscape of Human-Food Interaction Research. Proceedings of the 19th International ACM SIGACCESS Conference on Computers and Accessibility - DIS '18 (Hong Kong, China, 2018), 243-248.

[2] Amores, J. and Maes, P. 2017. Essence: Olfactory Interfaces for Unconscious Influence of Mood and Cognitive Performance. Proceedings of the 2017 CHI Conference on Human Factors in Computing Systems Pages 28-34 (2017), 28-34.

[3] Berenstein, N. 2018. Flavor Added: The Sciences of Flavor and the Industrialization of Taste in America. University of Pennsylvania.

[4] Brooks, J. et al. 2020. Trigeminal-based Temperature Illusions. Proceedings of the SIGCHI Conference on Human Factors in Computing Systems (CHI'20). (2020).

[5] Carr, M. et al. 2020. Dream engineering: Simulating worlds through sensory stimulation. Consciousness and Cognition. 83, (Aug. 2020), 102955. DOI:https://doi.org/10.1016/j.concog.2020.102955.

[6] Chamberlain, S. et al. 2020. rcrossref: Client for Various "CrossRef" "APIs."

[7] Clepper, C. 2016. The Rigged House: Gimmickry, Exhibition, and Embodied Spectatorship in Mid-Century American Movie-Going. Northwestern University.

[8] Dahl, R. 1964. Charlie and the Chocolate Factory. George Allen \& Unwin.

[9] Flouris, A.D. and Schlader, Z.J. 2015. Human behavioral thermoregulation during exercise in the heat: Human behavioral thermoregulation. Scandinavian fournal of Medicine \& Science in Sports. 25, (Jun. 2015), 52-64. DOI:https://doi.org/10.1111/sms.12349.

[10] Gayler, T. et al. 2020. Material Food Probe: Personalized 3D Printed Flavors for Emotional Communication in Intimate Relationships. Proceedings of the 2020 ACM Designing Interactive Systems Conference (Eindhoven Netherlands, Jul. 2020), 965-978.

[11] Han, T. et al. 2020. Mouillé: Exploring Wetness Illusion on Fingertips to Enhance Immersive Experience in VR. Proceedings of the 2020 CHI Conference on Human Factors in Computing Systems (Honolulu HI USA, Apr. 2020), 1-10.

[12] Heilig, M. 1962. Sensorama simulator. US3050870A. Aug. 28, 1962.

[13] IJzerman, H. and Semin, G.R. 2010. Temperature perceptions as a ground for social proximity. Journal of Experimental Social Psychology. 46, 6 (Nov. 2010), 867-873. DOI:https://doi.org/10.1016/j.jesp.2010.07.015.

[14] Iwata, H. et al. 2004. Food simulator: a haptic interface for biting. (2004), 51-57.

[15] Laube, H. Motion pictures with synchronized odor emission. US2813452A.

[16] Lin, Y.-J. et al. 2020. FoodFab: Creating Food Perception Illusions using Food 3D Printing. Proceedings of the 2020 CHI Conference on Human Factors in Computing Systems (Honolulu HI USA, Apr. 2020), 1-13.

[17] Matsukura, H. et al. 2017. Tracking of a Gas Plume With the Aid of Olfactory Assist Mask. IEEE Sensors fournal. 17, 16 (Aug. 2017), 5332-5340. DOI:https://doi.org/10.1109/JSEN.2017.2721968.

[18] Matsukura, H. and Ishida, H. 2018. Review on Development of Devices for Amplifying Human Olfaction: Approaches using Real and Virtual Concentration Method. IEEf Transactions on Sensors and Micromachines. 138, 8 (Aug. 2018), 337-342. DOI:https://doi.org/10.1541/ieejsmas.138.337.

[19] Obrist, M. et al. 2014. Temporal, affective, and embodied characteristics of taste experiences: a framework for design. (2014), 2853-2862.

[20] Obrist, M. et al. 2016. Touch, Taste, \& Smell User Interfaces: The Future of Multisensory HCI. Proceedings of the 2016 CHI Conference Extended Abstracts on Human Factors in Computing Systems - CHI EA '16 (San Jose, California, USA, 2016), 3285-3292.

[21] Peiris, R.L. et al. 2019. ThermalBracelet: Exploring Thermal Haptic Feedback Around the Wrist. Proceedings of the 2019 CHI Conference on Human Factors in Computing Systems - CHI '19 (Glasgow, Scotland Uk, 2019), 1-11.

[22] Peiris, R.L. et al. 2017. ThermoVR: Exploring Integrated Thermal Haptic Feedback with Head Mounted Displays. Proceedings of the 2017 CHI Conference on Human Factors in Computing Systems - CHI '17 (Denver, Colorado, USA, 2017), 5452-5456.

[23] Ranasinghe, N. et al. 2019. Augmented Flavours: Modulation of Flavour Experiences Through Electric Taste Augmentation. Food Research International. 117, (Mar. 2019), 60-68. DOI:https://doi.org/10.1016/j.foodres.2018.05.030.

[24] Ranasinghe, N. et al. 2018. Season Traveller: Multisensory Narration for Enhancing the Virtual Reality Experience. Proceedings of the 2018 CHI Conference on Human Factors in Computing Systems (2018), 1-13.

[25] Spence, C. et al. 2017. Digitizing the chemical senses: Possibilities \& pitfalls. International fournal of Human-Computer Studies. 107, (Nov. 2017), 62-74. DOI:https://doi.org/10.1016/j.ijhcs.2017.06.003.

[26] Stevenson, R.J. 2010. An Initial Evaluation of the Functions of Human Olfaction. Chemical Senses. 35, 1 (Jan. 2010), 3-20. DOI:https://doi.org/10.1093/chemse/bjp083.

[27] Sutherland, I. 1965. The Ultimate Display. Proceedings of the IFIP Congress (1965), 506-508.

[28] Vi, C.T. and Obrist, M. 2018. Sour Promotes Risk-Taking: An Investigation into the Effect of Taste on Risk-Taking Behaviour in Humans. Scientific Reports. 8, 1 (Dec. 2018), 7987. DOI:https://doi.org/10.1038/s41598-018-26164-3.

[29] 1952. Substance X. Tales of Tomorrow. 